\title{
Sulla risposta elastica delle dighe e sulla sua variazione nel tempo
}

\author{
(On the dams elastic reaction and its variation in the long run) \\ P. Caloi - M. C. Spadea
}

Ricevuto il 10 Gennaio 1969

Riassunto. - Vengono esaminate le reazioni di una diga, o di sue parti, alle sollecitazioni elastiche. Si espongono i concetti di effello di conrogliamento e di effelto di superficie e se ne valutano le entità in alcuni casi. Si accenna allazione di diffusione dell energia elastica, in corrispondenza delle superficie di discontinnitit dinamica fra concio e concio. Si esamina il comportamento di una diga, come un tutto vibrante. Si discutono le particolari caratteristiche della propagazione delle onde elastiche, lungo i conci di una diga.

SUMARY. - The reactions to elastic strains of a dam or any of its parts are being examined, the notions of conveyance effect and surface effect defined and their values in some cases estimated. Indications are given on the action of the distribution of the elastic energy, correlative to the surfaces of dynamic discontinuity between the varions concrete slabs. The behaviour of a dam taken as a vibrating unity is examined and peculiarities of elastic wave propagation along the concrete slabs of a dam are discussed.

The occurrence in a dam of the following, contradicting phenomena is proverl.

1. - Upwards, along a single concrete slah, there results a concentration of energy towards the crown, the more marked as the thinning of the concrete slah towards the top is more pronounced. This concentration of energy, which translates itself into an increase of the displacements associated with elastic waves, we deseribe as with the qualification Conveyance effect.

2. At the surface of the terminal passageway on top of a dam, there appears a further increase of the displacements (similar to the one observed with ordinary seismic waves at the surface of indefinite media). This wonld be the phenomenon known as surface effect.

3. - Transversely to the dam, at the equal level from right to left (or vice versa), there appears instead a noticeable distribution of elastic energy correlative to the surface of dynamic discontinnity interposed be- 
tween concrete slabs. The progressive extinction of energy in this transverse propagation seems to be due, for its greatest part, to reflection phenomena correlative to the named surfaces.

4. - Besides conducing elastic energy, the concrete slahs of a dam are apt to become the seat of oscillations of their own, characterized hy periods of oscillation definitely higher than the ones associated with the longitudinal and transverse waves conduced by the medium.

5. - The whole dam may settle into free oseillation, in case of strains or stresses apt to invest it in its totality, as usually happens on opening the bottom or half-bottom ontlets of the dam. The oscillations show evidently the highest periods in the capacity of the structure (for instance, in case of the Pieve di Cadore-dam, of the order of $1,5 \mathrm{sec})$.

6. - The propagation of body waves, longitudinal or transverse, when occurring in definite media, is realized as if the medium was extremely rigidel $(\sigma=0)$.

This summarized exposé refers to the reaction of the dam to a sudden alteration of the elastic field. Numerous studies have been already dedicated to gradual alterations - determinable especially with clinographs - as associated with thermic, climatic, gravimetric, tectonic and other phenomena.

1. - Un problema, che merita considerazione, è quello del trasferimento dell'energia elastica dalla base del tampone al coronamento di una diga. Esso può essere considerato sotto due aspetti:

1) Trasferimento sotto forma di oscillazioni libere. Tali oscillazioni, qualunque sia la loro nodalita, presenteranno i massimi spostamenti nel coronamento e - generalmente - presenteranno componenti verticali e spalla-spalla di trascurabile ampiezza. Di esso si è ampiamente discusso in precedenti pubblicazioni. Ulteriori considerazioni saranno svolte ai nn. 3 e 4.

2) Trasferimento sotto forma di oscillazioni longitudinali e trasversali nel mezzo. Indichiamo con e l'energia, associata a vibrazioni spaziali, longitudinali o trasversali, convogliate - in un determinato istante - alla base di un concio della diga.

Siano $e_{1}, e_{2}$ le densità di energia trasferite in due sezioni orizzontali del concio, di superficie $s_{1}, s_{2}$, alle quote $q_{1}, q_{2}$. Prescindendo dalla dissipazione nel mezzo, avremo

da cui

$$
e=e_{1} s_{1}=e_{2} s_{2} \text {. }
$$

$$
e_{2}=e_{1} \frac{s_{1}}{s_{2}}
$$


Poiché uno dei due lati delle sezioni rimane pressochè costante, al variare della quota - e precisamente il lato trasversale - se indichiamo con $l_{1}, l_{2}$ gli spessori del concio alle quote $q_{1}, q_{2}$, la [1] può scriversi:

$$
e_{2}=e_{1} \frac{l_{1}}{l_{2}} .
$$

Per vibrazioni coperiodali, le energie sono proporzionali ai quadrati degli spostamenti. Indicando con $a_{1}, a_{2}$ le ampiezze osservate alle quote $q_{1}, q$, avremo pertanto:

$$
a_{2}=a_{1} \sqrt{\frac{l_{1}}{l_{2}}} .
$$

Se una delle registrazioni avviene alla superficie del concio sul coronamento - sarà d'uopo tener conto anche dell'effetto di superficie (per onde longitudinali o trasversali). Quindi, se indichiamo con $f$ il fattore corrispondente all'effetto accennato, e se la quota $q$, è quella del coronamento, ivi potremo osservare, con buonissima approssimazione:

$$
a_{2}=a_{1} \sqrt{\frac{l_{1}}{l_{2}}} \cdot f .
$$

Per quote intermedie, vale la [3].

L'ampiezza delle registrazioni al coronamento assomma anche l'effetto di superficie. La [4] ei consente di ottenere tale efietto, ricavando $f$ :

$$
f=a_{2} / a_{1} / \overline{\frac{i_{2}}{l_{1}}},
$$

con manifesto significato dei simboli.

In sismologia, generalmente, il valore di $f$ è dell'ordine di 2. Tale valore però è calcolato sulla base della teoria dei raggi sismici e risulta soddisfatto, con buona approssimazione, solo per apprezzabili lunghezze d'onda. Una lacuna della teoria, infatti, è che, nei raggi sismici, si prescinde dalla lunghezza d'onda del fronte, al quale il raggio sismico è associato. ì noto d'altronde che, nell'ambito delle altissime frequenze - e quindi delle piccole lunghezze d'onda la propagazione delle onde elastiche si presenta, sotto molti riguardi, fortemente anomala. Basterà citare, a questo proposito, il fenomeno della dispersione anomala, scoperto da Caloi (1). L'osservazione ha provato che fortemente anomalo si presenta, sempre nel campo delle microlunghezze d'onda, anche l'effetto di superficie specialmente in 
un intorno di vibrazioni con periodi dell'ordine di 0.2 sec. In registrazioni sismiche ottenute in miniera e sulla superficie esterna, ricercatori giapponesi hamno ottenuto per $f$, per oscillazioni associate a periodi dell'intorno su detto, valori variabili fra 4 e 16 , mentre per vibrazioni piì rapide, l'effetto torna verso valori normali. Queste indagini, ottenute per-mezzi indefiniti, possono senz'altro essere paragonate a quelle di cui qui si parla — a quanto ('i risulta, efiettuate per la prima volta —, che riguardano la superficie di mezzi ben delimitati.

Pertanto, presenta notevole interesse il controllo dell'eventuale parallelismo che l'effetto di superficie presenta in mezi indefiniti e in mezzi ben delimitati.

A mò d'esempio, per la diga del Lumiei (presso Sauris), l'efietto di convogliamento fra le quote $q_{1}=896 \mathrm{~m}$ e $q_{2}$ (coronamento) $=98 \mathrm{~mm}$ risulta pari a 1.66, essendo - alle quote considerate - gli spessori rispettivamente:

$$
l_{1}=\mathrm{m} 11 ; \quad l_{2}=\mathrm{m} 4 .
$$

Per valutare il fattore espressione dell'efletto di superficie, sono state prese in considerazione le registrazioni ottenute alle quote $q_{1}$ e $q_{2}$ di piccole esplosioni effettuate sul fondo valle, in destra e in sinistra della linea di valle.

Il fattore $\left(l_{2} / l_{1}\right)^{1 / 2}$, ovviamente, per entrambe le registrazioni, ì uguale a 0.603: i rupporti fra le ampiezze lette sui vibrogrammi corrispondenti alle suddette registrazioni, sono rispettivamente uguali a 4 e 4.17. Sostituendo questi valori nella [4] si è avuto per il fattore $f$, dallo seoppio in destra,

dallo scoppio in sinistra,

$$
f=2.41
$$

$$
f=2.50 \text {. }
$$

Possiamo concludere che l'efletto di superficie - come ci si poteva attendere, data l'omogeneità della diga in esame - risulta pressochè uguale sia che si registrino seoppi provenienti dalla destra della roccia di fondo, sia che si registrino spari provenienti dalla sinistra.

Come ulteriore applicazione, riportiamo i risultati delle indagini, condotte presso la diga dell'Ambiesta (Tolmezzo), fra le quote dove si osservano i valori $l_{1}$ (coronamento) $=\mathrm{m} 2.1, l_{2}=\mathrm{m} 5.6$, per gli spessori in corrispondenza del concio centrale.

Tali indagini, condotte nella prima decade del Novembre 1967 , ebbero Io scopo di valutare l'eftetto di convogliamento del concio centrale della diga, nei confronti di gamme d'onda diverse; confron- 
tare tale effetto con quello di superficie, determinato in precedenti indagini; nonché valutare, per la prima volta, il coefficiente di estinzione dell'energia elastica - associata ad elevate frequenze - in corrispondenza delle superficie di discontinnità dinamica, intercedenti tra concio e concio.

\section{AMBIESTA \\ Novembre 1967 \\ R. SOd}

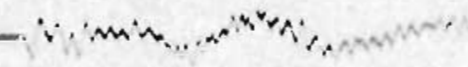

Fig. I - Esempio di effetto di convogliamento: registrazioni ottenute nel cunicolo sotto il coronamento $\left(R_{0} S_{D 1}\right)$ e sul tampone $\left(R_{T} S_{8 D}^{\prime}\right)$.

Nielle esperienze effettuate nel Novembre 1967, le registrazioni furono eftettuate alla base del concio centrale (tampone) e dentro il cunicolo sottostante al coronamento, entrambe in corrispondenza delle postazioni clinografiche ivi funzionanti (Fig. 1 e 2). Le registrazioni in quota, essendo realizzate qualche metro al di sotto del piano stradale, 
con cui culmina la diga, avevano lo scopo di sottrarsi all'eftetto di superfieie; proposito questo reso ragionevole dalla estremamente piccola lunghezra d'onda, associata alle vibrazioni longitudinali.

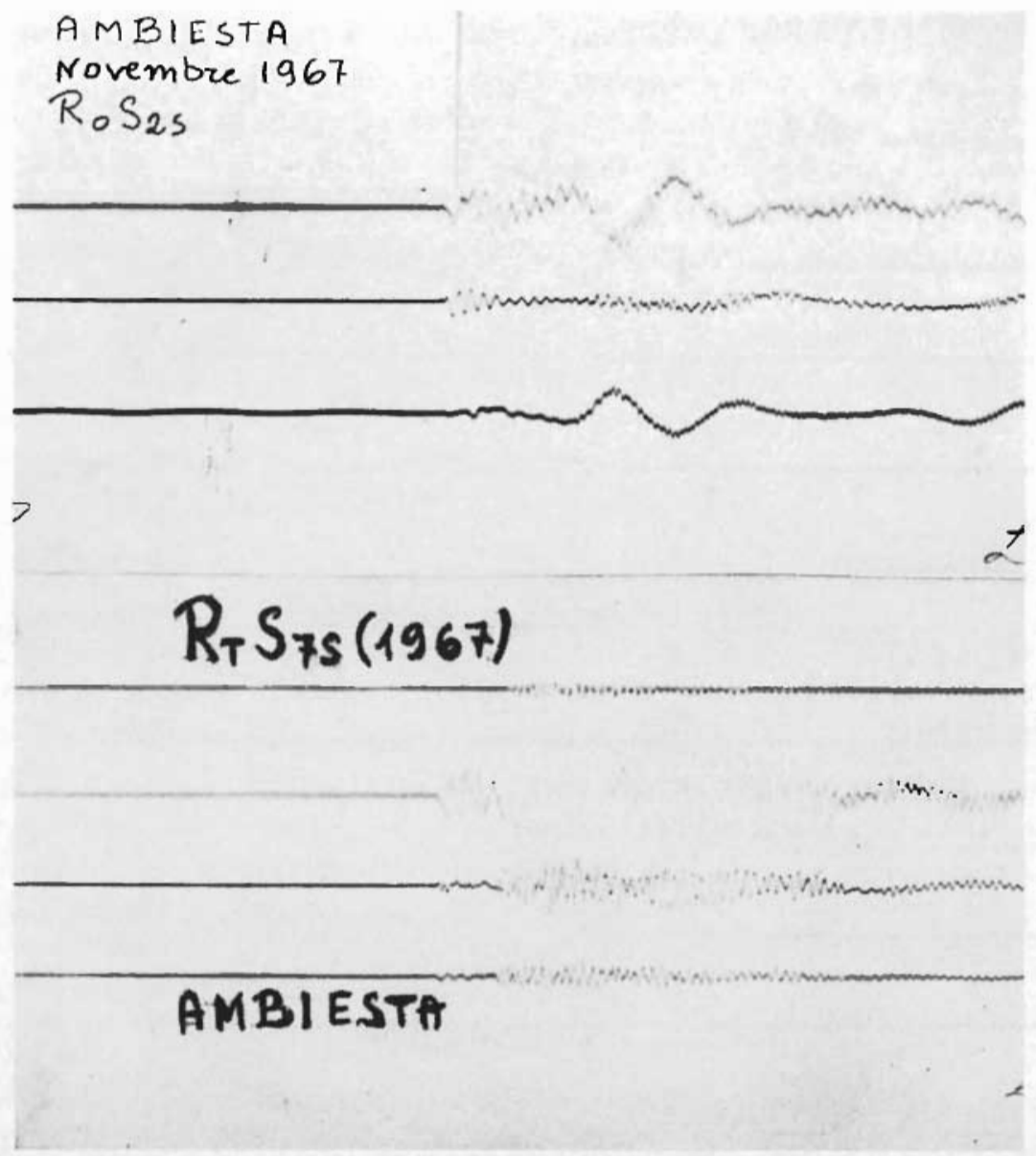

Fig. 2 - Esempio di convogliamento: registrazioni ottenute nel cunicolo sotto il coronamento $\left(R_{9} S_{2 s}\right)$ e sul tampone $\left(R_{T} S_{7 s}\right)$.

Per i valori di $l_{1}, l_{2}$ sopra riportati, l'effetto di convogliamento fra le due postazioni di registrazione doveva risultare dell'ordine di 1.63. Ricordiamo che l'effetto di superficie (se fosse stato ancora presente entro il cunicolo sotto il coronamento), si doveva ottenere dall'applicazione della [5]. 
Ebbene, la media di tutti i valori ottenuti è risultata di

$$
0.97 \text {; }
$$

risultato davvero eccellente, attesa lapprossimazione che ci si deve aspettare in queste esperienze. Se sotto il coronamento, presso la postazione clinografica, l'efletto di superficie fosse stato nullo, la [5] avrebbe dovnto assumere il valore 1. E tale valore nelle esperienze esperite, come si è visto, di fatto assume. Basti osservare che i valori più discosti dall'unità furono 0.85 in difetto e 1.16 in eccesso.

Resta cosi confermata la validità dei risultati ottenuti nelle esperienze dell'anno precedente. Pertanto l'efletto di superficie, che si ottiene registrando sul piano stradale della diga (in corrispondenza del concio centrale), è dell'ordine di

\subsection{0}

essendosi ivi ottenuto $a_{2} / a_{1}=2.75$, sulla base delle registrazioni avute in quella occasione.

Le esperienze eseguite con geofoni - che sostanzialmente confermano, in media, quelle condotte con vibrometri -, non presentano, in ogni modo, sufficiente attendibilita, mancando la taratura dei singoli geofoni.

2. - Per calcolare la diffusione dell'energia elastica, in corrispondenza delle superficie separanti un concio dai conci limitrofi, si sono effettuate registrazioni con vibrografi, posti agli estremi sinistro e destro del cunicolo di coronamento, di piccole esplosioni provocate in roccia, a contatto di detto cunicolo, nel suo estremo destro.

I risultati ottenuti, come si è accennato, presso la diga dell' $\mathrm{Am}$ biesta non potevano essere più significativi. Ia perdita di energia nella propagazione da destra a sinistra, a quota cunicolo di coronamento, è notevole. Basti osservare che l'ampiezza delle onde longitudinali, si riduce di almeno $1 / 24$. Poiché, a parità di altre condizioni (nel nostro caso, a parità di frequenza), l'energia di un'onda piana varia proporzionalmente al quadrato dell'ampiezza, possiamo ritenere che l'energia uscente in sinistrà è circa 1/600 dell'energia entrante, alla stessa quota, in destra (all'altezza del cunicolo di coronamento). In realtà, il treno d'onda entrante dalla destra può ritenersi esaurito all'estrema sinistra della diga. Ciò, del resto, in conformità della teoria.

Tale perdita va, nella quasi toialità, attribuita a fenomeni di riflessione dell'energia elastica, in corrispondenza delle superficie di 
discontinuità dinamica, separanti i vari conei fra loro. Si ritiene qui opportuno un richiamo sulla teoria delle onde riflesse e rifratte.

Indichiamo con $P$ le onde longitudinali, con $S$ le onde trasversali, con $S V$ le onde trasversali oscillanti nel piano di propagazione e $S H$ le analoghe tangenziali. Per le altre grandezze, valgano inoltre i seguenti simboli:

Radice quadrata rapporto d'energia $F$ Angolo d'incidenza

Velocità

Densità

Rigidità

Rapporto di Poisson

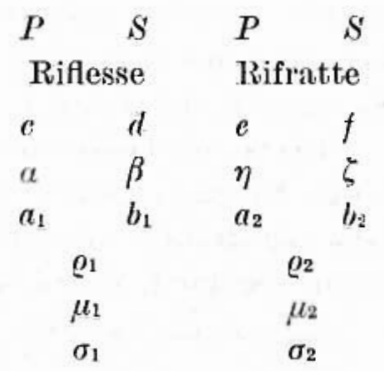

$$
\begin{aligned}
& \gamma=\mid \frac{\overline{\varrho_{1}}}{\varrho_{2}}, n=\frac{a_{2}}{a_{1}}, q=\frac{a_{1}}{b_{1}}, p=\frac{b_{2}}{b_{1}}, \\
& r=\frac{b_{2}}{a_{1}}=\frac{p}{q}, s=\frac{a_{2}}{b_{1}}=n q, t=p q .
\end{aligned}
$$

Vanno richiamate le formule:

$$
\sigma_{1}=\frac{1}{2}\left(1-\frac{1}{q^{2}-1}\right),
$$

e analogamente per $\sigma_{2}$ :

$$
\begin{gathered}
\sin \alpha: \sin \beta: \sin \eta: \sin \zeta=a_{1}: b_{1}: a_{2}: \dot{o}_{2}, \\
c^{2}+d^{2}+e^{2}+f^{2}=1 .
\end{gathered}
$$

Partendo dalle equazioni fondamentali, relativi alle onde riflesse e rifratte, se I'onda incidente è una $P$, le radici quadrate dei rapporti d'energia sono date da $\left({ }^{2}\right)$ :

$$
\begin{aligned}
& 1+e+I d-j J e+j K f=0 \\
& 1-c+\frac{1}{I} d-\frac{j}{J} e-\frac{j}{K} f=0 \\
& -1+c+L d+\frac{p}{\jmath} \frac{1}{J} e-\frac{t}{j} Q f=0 \\
& -1-c+\frac{1}{L} d+\frac{1}{j} J N e+\frac{r}{j} R f=0 .
\end{aligned}
$$


Analogamente, per un'onda incidente ST:

$$
\begin{aligned}
& -1+d-I e-j \frac{I}{K} f-j \frac{I}{J} e=0 \\
& 1+d+\frac{1}{I} e+j \frac{K}{I} f-j \frac{J}{I} e=0 \\
& 1+d-L e+\frac{p^{2}}{j} \frac{K}{I} f+\frac{s}{j} U e=0 \\
& -1+d+\frac{1}{L} e-\frac{1}{j} \frac{I}{K} N f+\frac{p^{2}}{j s} \frac{N}{U} e=0 .
\end{aligned}
$$

Nei due gruppi di equazioni ̀̀ $(*)$ :

$$
\begin{aligned}
& I=\sqrt{\operatorname{cotg} \alpha \cdot \operatorname{cotg} \beta} ; \quad J=\sqrt{\operatorname{cotg} \alpha \cdot \operatorname{tang} \eta} ; \quad K=\sqrt{\operatorname{cotg} \alpha \cdot \operatorname{cotg} \zeta} ; \\
& \frac{I}{K}=\sqrt{\operatorname{cotg} \beta \cdot \operatorname{tang} \zeta} ; L=q \sqrt{\frac{\operatorname{cotg} 2 \beta \cdot \cos 2 \beta}{\sin 2 \alpha}}=\frac{\operatorname{cotg}^{2} \beta-1}{2 I} ; \\
& N=\frac{\cos 2 \zeta}{\cos 2 \beta} ; \quad Q-\sqrt{\frac{\operatorname{cotg} 2 \zeta \cdot \cos 2 \zeta}{\sin 2 \alpha}} ; \quad I=\frac{\sqrt{\sin 2 \zeta \cdot \sin 2 \alpha}}{\cos 2 \beta} ; \\
& U=\frac{\cos 2 \zeta}{\sqrt{\sin 2 \beta \cdot \sin 2 \eta}} .
\end{aligned}
$$

Se l'onda incidente è un'onda $S H$, si può avere solo un'onda riflessa ed eventualmente rifratta, $S H$. Le radici quadrate del loro rapporto d'energia sono date da:

$$
d=\frac{1-H}{1+H}, f-\frac{2}{1+H} \mid \bar{H},
$$

dove

$$
H=\frac{\varrho_{2} b_{2}}{\varrho_{1} b_{1}} \cdot \frac{\cos \zeta}{\cos \beta}-\frac{\varrho_{2} \sin 2 \zeta}{\varrho_{1} \sin 2 \beta}-\frac{\mu}{\mu_{1}} \cdot \frac{\operatorname{cotg} \zeta}{\operatorname{cotg} \beta} .
$$

Nelle precedenti equazioni il segno positivo caratterizza un'onda longitudinale corrispondente ad un movimento nella direzione fra il

$\left({ }^{*}\right)$ Gitenberg, a coefficiente di ${ }_{j}^{*}$ e nella $3^{\text {a }}$ delle [6] pone la lettera $M$, alla quale attribuisce il valore $u=\frac{\sqrt{\cos n}}{\cos \alpha}$; in realtà è invece,$U=\frac{1}{J}=$ botg $\eta$ tang $u$. Inoltre, se le [7] sono dedotte dalle corrispondenti equazioni di Knott, in esse l'unità - che costituisce il primo termine - andrebbe in tutte cambiata di segno. 
mezzo dell'onda incidente a quello dell'onda rifratta. Nelle onde rifratte $P$, il segno positivo indica che un'onda (incidente) di rompressione è trasmessa come compressione, oppure che una dilatazione conserva il suo carattere anche dopo la rifrazione; un mutamento di segno corrisponde ad uno scambio fra compressione e dilatazione o viceversa.

I risultati per casi speciali (come per es. $\alpha=0, \beta=0, \alpha=90^{\circ}$, $\beta=90^{\circ}$ ) conseguono agevolmente dalle equazioni.

Generalmente, la densità come le costanti elastiche di una sostanza cristallina sono maggiori delle corrispondenti dello stesso materiale ad una temperatura superiore al suo punto di fusione. Benchè i dati sperimentali per rocee dell'interno della Terra, alla profondità dove questo punto di fusione è raggiunto, siano del tutto mancanti, Gutenberg ritiene utile considerare la possibilità ('he il rapporto delle due densità sia pressochè uguale a quello delle costanti elastiche e che la variazione nella velocità dell'onda fra i due stati sia piccolo. Le equazioni corrispondenti a questo caso speciale conseguono dalle $[6],[7]$.

Per un'onda $P$ incidente, si ha:

$$
\begin{gathered}
1+c+I d-j e-j I f=0 \\
1-c+\frac{1}{I} d-j e-\frac{j}{I} f=0 \\
-1+e+L d+\frac{1}{j} e-\frac{I}{j} f=0 \\
-1-e+\frac{1}{L} d+\frac{1}{j} e+\frac{1}{j L} f=0 .
\end{gathered}
$$

Le corrispondenti equazioni per un'onda incidente $S V$ sono:

$$
\begin{aligned}
& 1-d+I c+j f+j I e=0 \\
& 1+d+\frac{1}{I} c+j f-\frac{j}{I} e=0 \\
& -1-d+L e-\frac{1}{j} f-L e=0 \\
& -1 \neg d+\frac{1}{L} c-\frac{1}{j} f+\frac{1}{j L} e=0 .
\end{aligned}
$$

Dal confronto dei sistemi [8] e [9] risulta che la distribuzione dell'energia nei due tipi d'onda è pressoché uguale. 
Dalle [9] si traggono facilmente le seguenti relazioni per i rapporti delle radici quadrate dell'energia per onde incidenti is ${ }^{* *}$ :

$$
\begin{aligned}
& \frac{c}{e}=-\frac{j I^{\prime}+\frac{1}{j} L^{\prime}}{I^{\prime \prime}-L^{\prime \prime}}, \frac{d}{e}=-\frac{1}{2^{-}} \frac{j I^{\prime} L^{\prime \prime}+\frac{1}{3} I^{\prime \prime} L^{\prime}}{I^{\prime \prime}-L^{\prime \prime}}, \\
& \frac{j}{e}=\frac{\jmath+\frac{1}{j}}{j-\frac{1}{j}} \cdot \frac{I L+1}{I-L}, I L+1=\frac{1}{2}\left(\operatorname{cotg}^{2} \beta+1\right) \text {, }
\end{aligned}
$$

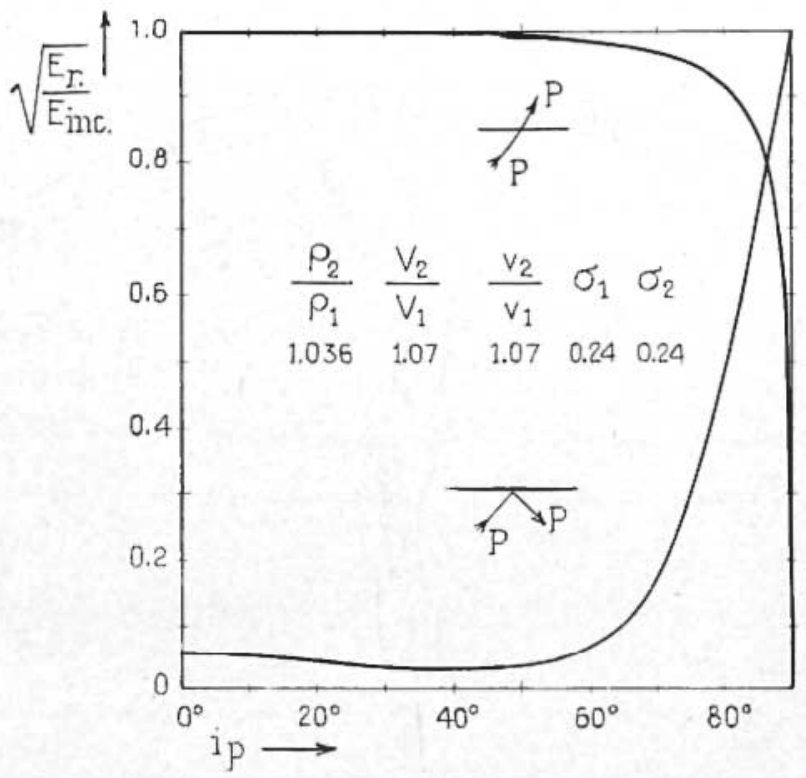

Fig. 3 - Curve clie danno le radici quadrate del rapporto fra energia riflessa o rifratta a quella incidente in funzione dell'angolo d'incidenza $i$, nel caso di incidenza su una discontinuità senza cambio di velocità (secondo (rutenberg).

(*) Nelle relazioni riportate da Gutenbergr (pag. 89 Nota citata) manca il segno meno davanti alle prime due, mentre nella terza - quella che dà il rapporto $/ / e$ - il primo $j$ a numeratore a a denominatore è sostituito dall unità. Questi ed altri errori figuranti nella nota di Gutenberg sull'argomento $\left({ }^{2}\right)$, furono fatti rilevare all Autore da Caloi, con lettera del 19 Dicembre 1946. Lillustre, compianto geofisico con lettera del 15 Gennaio 1947 (che Caloi conserva), ebbe a riscontrare esatte le correzioni apportate. In letta lettera, Gutenberg concludeva con questa constatazione: "It is peculiar that these equations rarely have been printed without misprints". 
essendo:

$$
I^{\prime}=I+\frac{1}{I} ; L^{\prime}=L+\frac{1}{L} ; I^{\prime \prime}=I-\frac{1}{I} ; L^{\prime \prime}=L-\frac{1}{\vec{L}} .
$$

Equazioni analoghe nel caso di onde incidenti $P$.

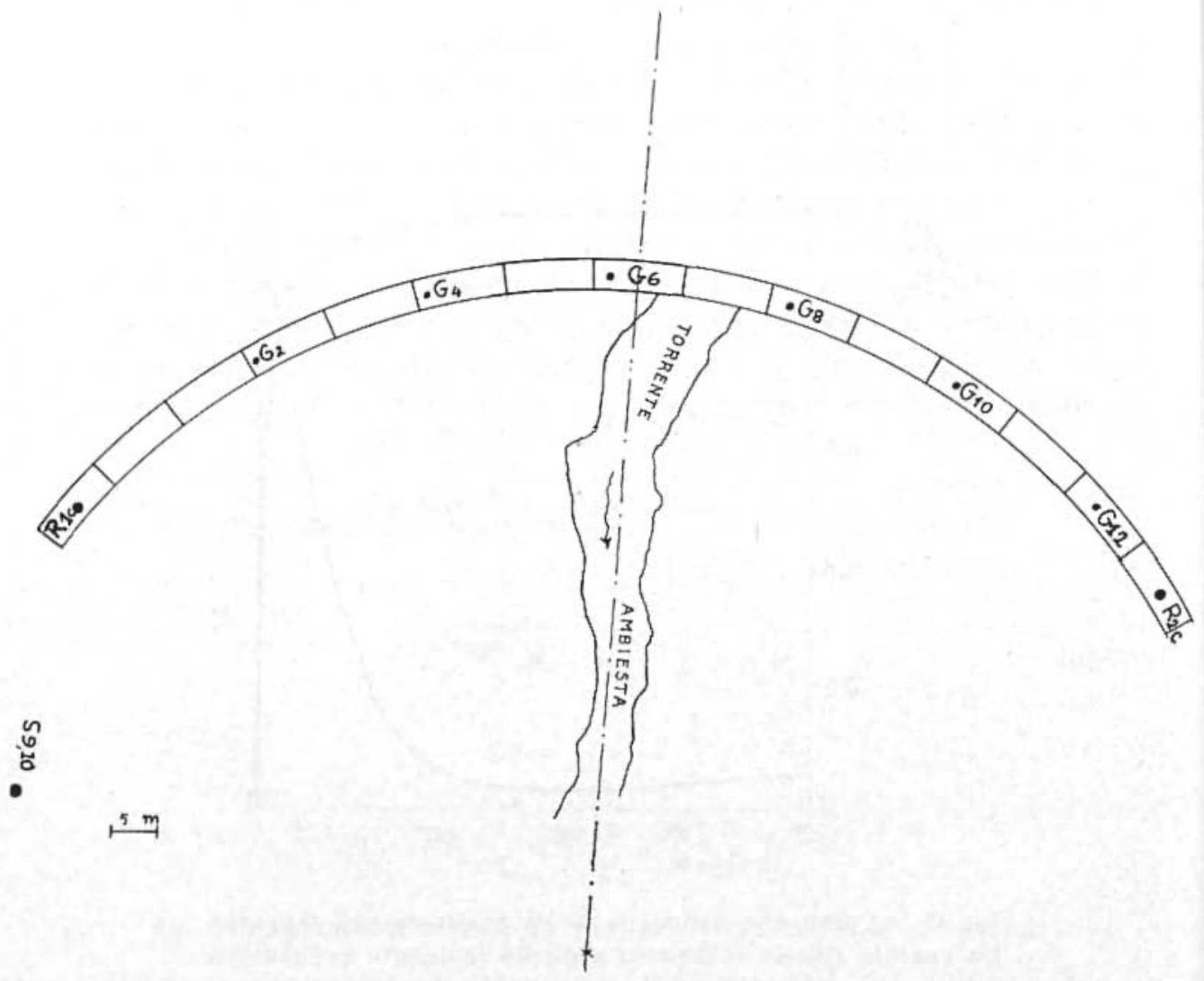

Fig. + - Rappresentazione schematica del coronamento diga dell Ambiesta, con la suddivisione in conci. $R_{1 c}$ ed $R_{2 c}$ si riferiscono alle postazioni del vibrometro; le $G$ alle postazioni dei geofoni: la $S$ ai punti di seoppio.

Nel nostro caso, interessa la distribuzione di energia per onde longitudinali incidenti in una discontinuità, senza variazione di velocità. Infatti, la discontinuità fra concio e concio è una discontinuità di natura esclusivamente dinamica, come quella che si verifica fra due mezzi, di uguali caratteristiche fisiche, messi a contatto lungo le loro superficie limiti. 
Diversi autori si cimentarono nella determinazione numerica della su detta suddivisione di energia, in casi diversi: Knott, Jeffreys, Blut, Schlichter e Gabriel, ecc. Gutenberg riuni i valori già calcolati e li
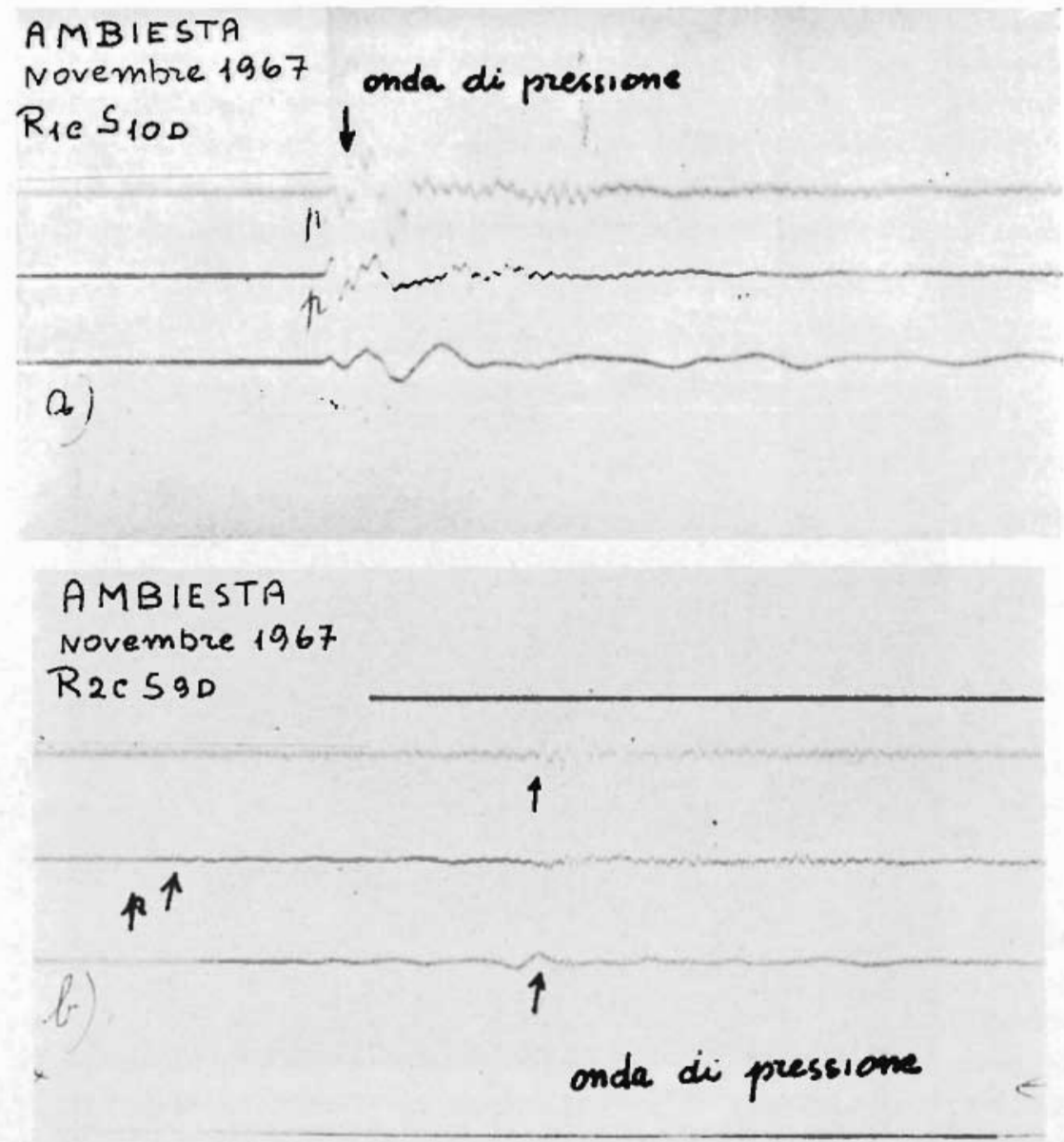

Fig. 5 - a) Registrazione ottenuta in $R_{2 c}$ (la $p$ sta per la $l$ lel testo); b) Registrazione ottenuta in $R_{1 c}$ all'estremo opposto del cunicolo. L'estinzione dell'energia, oltre ai fenomeni di riflessione in corrispondenza dei conci, è in parte da attribuire a diffusione. in quanto la propagazione avviene in una zona attraversata da un cunicolo.

completò. Riportiamo qui i relativi grafici, limitatamente ai casi che presentano analogia con la propagazione considerata fra concio e 
concio, e per i valori di angoli d'incidenza entro i limiti consentiti dal trasferimento dell'energia da un lato all'altro della diga (Figr. 3).

Si noti che il tragitto, da un estremo all altro, subisce una deviazione di $110^{\circ}$ (a (Fig. 4). Da un estremo all'altro intercedono una quindicina di superficie riflettenti: ammessa la perdita di energia dell'ordine di $1 / 10$ in corrispondenza di ogni superficie, resta giustificata l'effettiva estinzione dell'energia elastica alla fine del tragitto (Fig. 5). Le vibrazioni che, nell'estremo sinistro, vengono registrate con ritardo sono da attribuirsi all'urto dell'onda di pressione acustica nel cunicolo di coronamento.

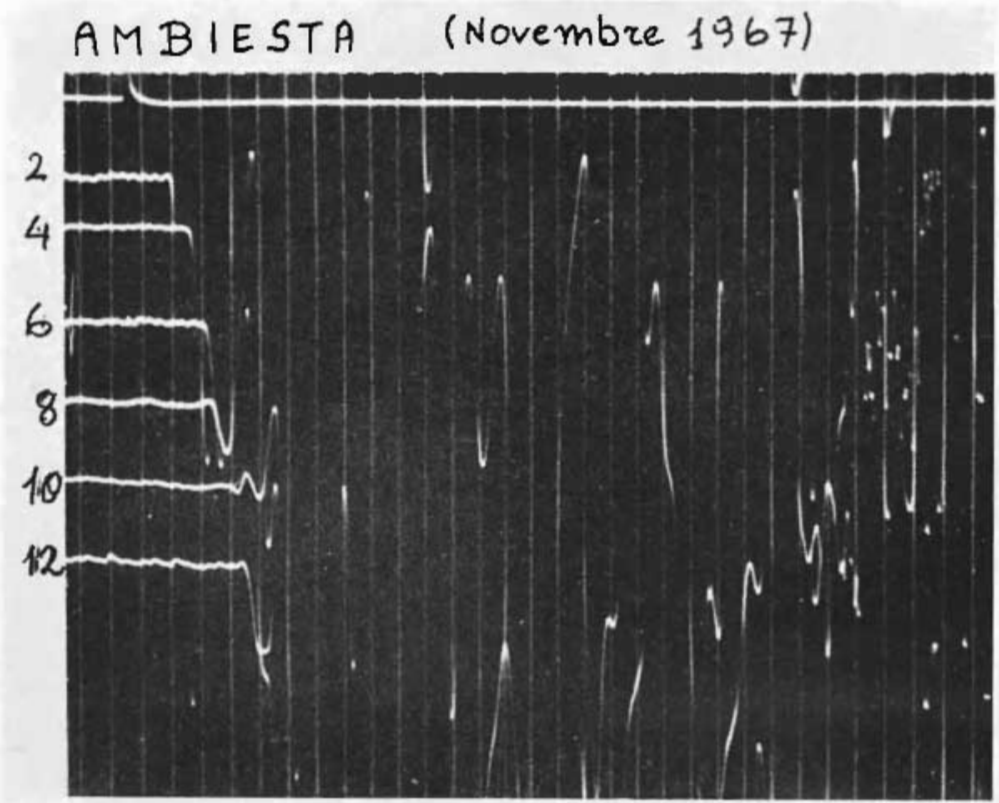

Fig. 6 - Sia pure qualitativamente anche i geofoni (distribuiti come da Fig. f) danno un ehiaro esempio di estinzione dell'energia elastica nel suo trasferimento da destra a sinistra. Vedi anche Figr. 5.

I geofoni, distribuiti a distanze crescenti da destra a sinistra, danno una visione qualitativa del fenomeno. Qualora essi fossero tarati, sarebbe possibile calcolare quantitativamente la progressiva riduzione di energia, nel suo trasferimento da destra a sinistra, associata alle alte frequenze delle onde longitudinali (V. Fig. 6).

3. - In relazioni e pubblicazioni precedenti, uno di noi ha esposto ed applieato un metodo per la determinazione delle vibrazioni proprie 
dei conei di una diga $\left({ }^{6}\right)$. Naturalmente non staremo a riesporre la relativa teoria, limitandoci a riportare la sola formula che dà il periodo proprio:

$$
T=\frac{4 \pi \mid \overline{3}}{\theta^{2}} \cdot \frac{l^{2}}{b} \cdot \frac{1}{\sqrt{\frac{E}{g}}},
$$

dove $l$ è la lunghezza del concio in esame, $b$ lo spessore medio, $\frac{t}{\varphi}$ la velocità delle onde longitudinali proprie del calcestruzzo di modulo $E$ (modulo di Young) e di densità $o$, e $\theta$ infine d̀ una delle radici della equazione:

$$
\cosh \theta+\cos \theta+1=0 \text {. }
$$

La formula riportata, come è già stato osservato, va intesa come formula di prima approssimazione. I motivi sono evidenti, essendo essa il risultato di schematizzazioni, d'altronde inevitabili. Va ancora aggiunto che i valori con essa ottenuti hamno significato solo per micromovimenti, durante i quali i vincoli fra concio e concio non influenzano in modo decisivo il periodo perseguito. Va inoltre sottolineato che i periodi osservati si ritengono relativi alle stesse condizioni dell'invaso, nel senso che grosse variazioni nel livello del bacino possono influire sui valori medi delle vibrazioni proprie dei conci della diga.

Ad ogni modo, l'interesse del metodo è legato alla sua ripetizione nel tempo - eseguita possibilmente, anzi necessariamente - nelle stesse condizioni, perchè non tanto il valore assoluto dei periodi conta (che può, più o meno in modo accentuato, discostarsi da quello teorico) quanto le sue eventuali variazioni nel tempo. Essenzialmente, a tali variazioni infatti, è legato il valore pratico del metodo stesso. Poichè gli elementi geometrici non mutano, le variazioni sono senz'altro da attribuirsi a mutamenti nella natura elastica del mezzo, mutamenti che non possono non tradursi in variazioni di periodo. 1'altronde, mutamenti nella densità sono generalmente trascurabili e comunque non sono mai tali da influire in modo determinante sulla dinamica del mezzo, la quale, invece, è strettamente legata al campo elastico. Intendiamo dire che eventuali variazioni del periodo sono soprattutto da imputare a variazioni nella grandezza $E$, ciò̀ a variazioni del modulo di elasticitì. 
Cosi, se $E_{1}$ ed $E_{2}$ sono i valori di $E$ (relativi al calcestruzzo) ottenuti in esperienze successive e $T_{1}, T_{2}$ i corrispondenti periodi osservati, a parità di altre condizioni, dalla [10] si ottiene:

$$
E_{2}=E_{1} \cdot\left(T_{1} / T_{2}\right)^{2}
$$

Ad esempio il calcestruzzo della diga del Lumiei in otto amni (1954-1962), avrebbe subito un irrigidimento dell'ordine di grandezza espresso da:

$$
E_{2}=E_{1} \cdot 1.34
$$

il periodo d'oscillazione libera essendo passato da $0^{8} .22$ del 1954 a 0 s.19 del 1962 .

Osserviamo che, almeno nei primi decenni, un irrigidimento del calcestruzzo delle dighe è stato generalmente notato anche attraverso altro genere di osservazioni, quale per esempio, la graduale attenuazione dell'onda diurna riscontrata presso le dighe di Pieve di Cadore, Ambiesta $\left({ }^{6}\right)$ ecc.

4. - Non sempre l'oscillazione propria registrata risulta da un impulso singolo, gradualmente smorzantesi, come generalmente si verifica invece per le oscillazioni proprie di isolati speroni di roceia nel loro ambiente naturale $\left(^{6}\right)$.

I legami esistenti fra concio e concio famo si che, anche per micromovimenti, le oscillazioni dei conci limitrofi possano interferire; in questo caso è ínevitabile la formazione di battimenti.

Eे noto che se indichiamo con $T_{1}$ e $T_{2}$ i periodi di due oscillazioni di ampiezza pressochè uguale $\left(T_{2}>T_{1}\right)$ la risultante è ancora un'oscillazione sinusoidale di periodo:

$$
p=\frac{2 T_{1} T_{2}}{T_{1}+T_{2}},
$$

la cui ampiezza varia con il periodo

$$
P=\frac{2 T_{1} T_{2}}{T_{2}-T_{1}} .
$$

La separazione dei due periodi avviene quindi mediante le formule:

$$
T_{1}=p P /(p+P) \quad T_{2}=p P /(P-p)
$$

dove $p$ e $P$ sono dati dalle osservazioni. 
Applicando la [11] ai dati sperimentali ottenuti presso le dighe di Lumiei, Barcis e Mis si sono avuti i seguenti risultati:

Tabella

\begin{tabular}{|c|c|c|}
\hline Dighe & $\begin{array}{c}T_{1} \\
\text { sece }\end{array}$ & $\begin{array}{r}T_{2} \\
\text { sec }\end{array}$ \\
\hline Lumiei (1965) & 0.13 & 0.18 \\
\hline Barcis (1964) & 0.08 & 0.11 \\
\hline$(196+)$ & 0.095 & 0.15 \\
\hline
\end{tabular}

Per la diga del Mis, oltre alle oscillazioni dei conci, si registrano anche quelle del ponte sovrastante il coronamento. Ciò è reso manifesto dalla cospicua componente verticale del movimento. Le oscillazioni si presentano sotto forma di battimenti: il periodo medio è dell'ordine del decimo di secondo (e nasce dalla combinazione di oscillazioni con periodi di $0.12 \mathrm{sec}$ e $0.084 \mathrm{sec}$, rispettivamente).

In tutte le dighe aventi il coronamento sovrastato da strutture a forma di ponte, si verifica la registrazione di oscillazioni, con periodi dell'ordine di quelli sopra riportati, sviluppate sopra tutto sulla componente verticale.

5. - In occasione dell'apertura degli scarichi di una diga, la struttura, come è noto, entra in vibrazione propria. In alcune dighe questa vibrazione raggiunge accentuate caratteristiche dinamiche (diga del Lumiei, p. es.).

Ia determinazione rigorosa, per via teorica, dei periodi propri di una diga - considerata come struttura vibrante - non è certo agevole. Basti pensare che, in realtà, una diga è un complesso di strutture, ciascuna con periodi propri, geometricamente asimmetriche, di spessori variabili; e le reciproche influenze variano non solo al variare del tipo di eccitazione, ma anche dell'intensità e del loogo di eccitazione.

D'altronde poiché in misure del genere è l'ordine di grandeza che conta, il perseguire soluzioni rigorose è spesso illusorio; diviene quindi preferibile ricorrere a schematizzazioni che, pur garantendo una sufficiente attendibilita, hamno il notevole vantaggio della semplicità e rapidità di applicazione. 
E quanto abbiamo ritenuto opportuno di fare nel caso specifico.

Una larvata approssimazione del periodo fondamentale di una diga, come mu complesso vibrante, può determinarsi considerando il manufatto come un arco circolare.

Già Horace Lamb, nel 1888, si era occupato - da par suo - delle vibrazioni di una sbarra curva $\left({ }^{3}\right)$. Il problema fu ripreso da Den Hartog, il quale, nel 1928, pubblicò un lavoro sulla più bassa frequenza naturale di archi cireolari, incardinati o fissati ai loro estremi, in presenza 0 in assenza di estensione del materiale $\left({ }^{4}\right)$.

Naturalmente, non ci soffermeremo su argomenti teorici. Ci limitiamo a riportare la formula che dà la pulsazione (1) del novimento, in funzione del modulo di Young $E$ del materiale vibrante, del momento d'inerzia $I$ della sezione trasversa della massa per unità di lunghezza della linea di centro $\gamma$ e del raggio $R$ dell'arco considerato.

Si laa (nel caso di inestensione) $\left(^{5}\right)$ :

$$
\omega=\frac{C_{3}}{\mathrm{R}^{2}} \sqrt{\frac{E I}{\gamma}},
$$

dove $C_{3}$ è dato dalla tavola:

$\begin{array}{ccccccccccc}\alpha \ldots & 0^{\circ} & 20^{\circ} & 40^{\circ} & 60^{\circ} & 80^{\circ} & 100^{\circ} & 120^{\circ} & 140^{\circ} & 160^{\circ} & 180^{\circ} \\ U_{3} \ldots & \infty & 504 & 124 & 53.8 & 29.2 & 17.9 & 11.85 & 8.22 & 5.93 & 4.38 \\ \alpha \ldots & 200^{\circ} & 220^{\circ} & 240^{\circ} & 260^{\circ} & 280^{\circ} & 300^{\circ} & 320^{\circ} & 340^{\circ} & 360^{\circ} \\ U_{3}^{\prime} . & 3.31 & 2.54 & 1.98 & 1.57 & 1.25 & 1.01 & 0.821 & 0.67 \tau & 0.56 \tau,\end{array}$

essendo a l'angolo al centro che sottende all'arco assegnato.

Dalla [12], se o esprime la densitì e $S$ l'area della sezione trasversa, si trae per il periodo:

$$
T=2 \pi \frac{R^{2}}{C_{3}} \frac{1}{v^{i} \frac{H}{\varrho}} \frac{1}{\frac{I}{S}} .
$$

Nel caso di sezione trasversa rettangolare, di lungezza $a$ e di larghezza $b$, si può scrivere:

$$
\sqrt{\frac{I}{S}}=\frac{\bar{b}}{2 \sqrt{3}} .
$$

Pertanto, sarà:

$$
T=\frac{4 \pi 1^{\prime 3}}{b} \cdot \frac{R^{2}}{C_{3}} \cdot \frac{1}{\sqrt{\frac{E}{\varrho}}} .
$$


Per la diga di Pieve di Cadore, per es., fatto $R=\mathrm{m} \mathrm{162,} \alpha=130^{\circ}$ - e quindi $C_{3}=10-, \frac{E}{\hat{E}}=5.000 \mathrm{~m} / \mathrm{sec}$, per $b=9 \mathrm{~m}$ risulta, all'incirca:

$$
T=1.26 \mathrm{sec} .
$$

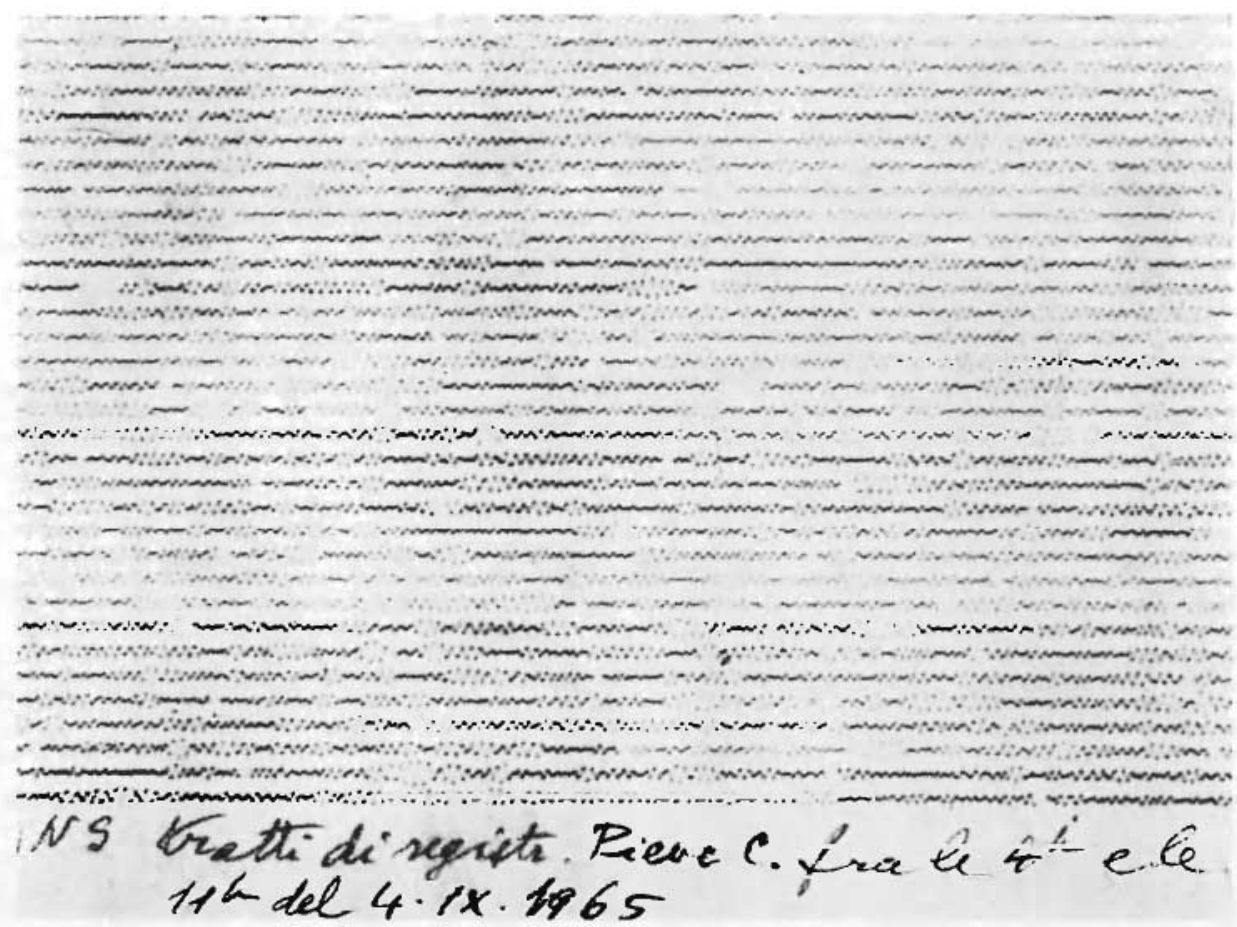

Fig. 7 - Regristrazioni di vibrazioni della diga di Pieve di C'adore, associate ad apertura degli searichi, a bacino pieno, registrate dalla stazione sismica sita in cabina comandi ad una quindicina di metri dalla diga.

Per la diga del Vajont (nelle attuali condizioni), fatto $R=90 \mathrm{~m}$, $b=6 \mathrm{~m}$, per $a=90^{\circ}\left(C_{3}=25\right)$, si ottiene:

$$
T=0.24 \mathrm{sec} \text {. }
$$

Con lo stesso valore per $\alpha$, fatto $R=100 \mathrm{~m} \mathrm{e} b=5 \mathrm{~m}$, risulta:

$$
T=0.35 \mathrm{sec} \text {. }
$$

Le osservazioni damno per la diga di Pieve di Cadore un valore medio di $1.2 \mathrm{sec}$. per il periodo, mentre per la diga del Vajont, si ottiene, in media, $T=0.4-0.5$ sec (Figg. 7,8 ). 
6. - E noto che, in un mezzo indefinito, di densità $\varrho$, caratterizzato da un modulo di Young $E$ e da un rapporto di Poisson $\sigma$, indicando con $v_{1}$ la velocità delle onde longitudinali, ̀̀:

$$
v_{1}=\sqrt{\frac{E}{\varrho} \frac{1-\sigma}{(1+\sigma)(1-2 \sigma)}} .
$$

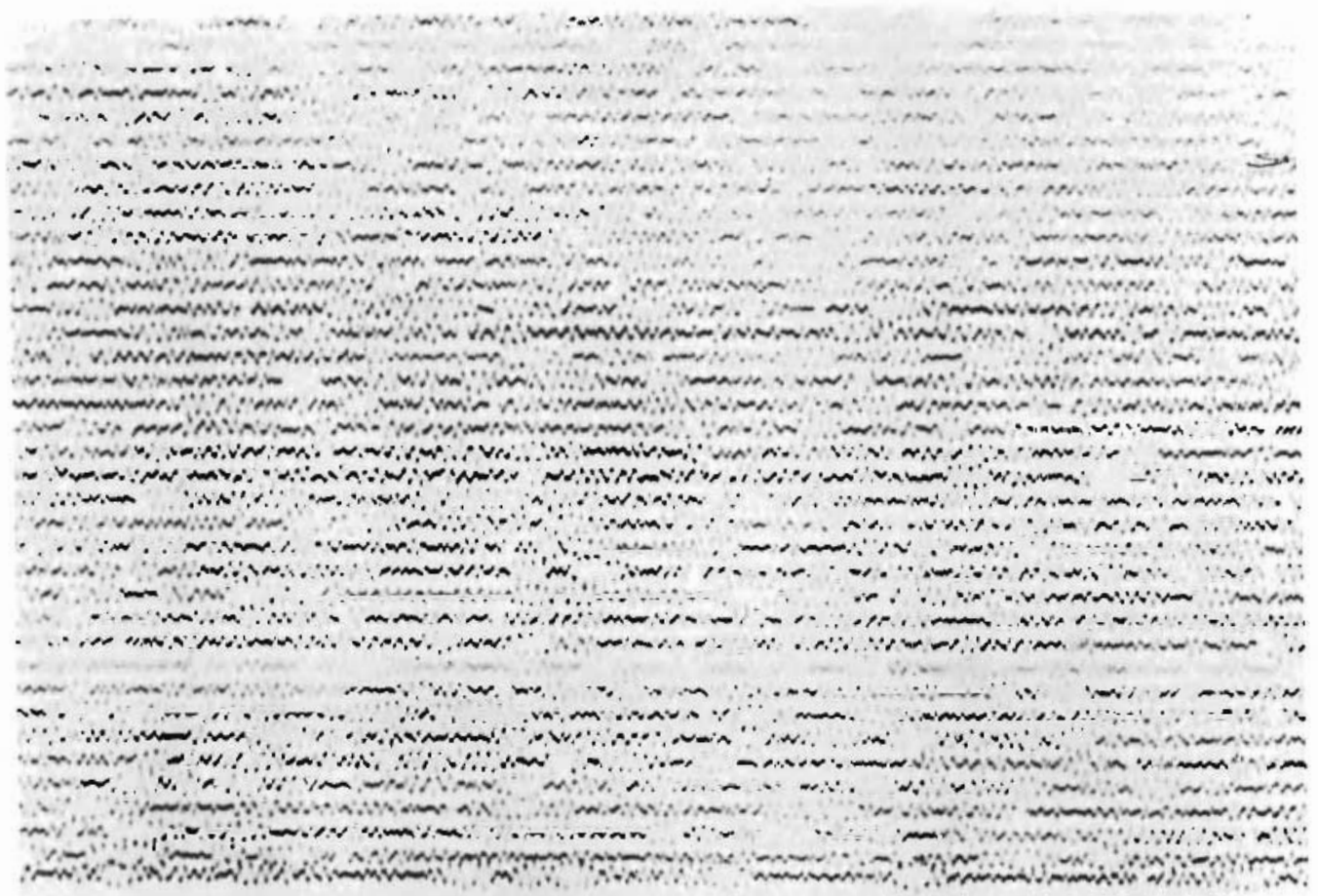

\section{Ew

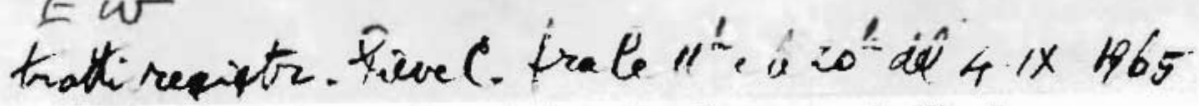

Fig. 8 - Esempi di registrazione come da Fig. 7 .

In un mezzo finito invece, la velocità delle onde longitudinali vale:

$$
v_{t}=\sqrt{\frac{E}{\varrho}} .
$$

Poiché

$$
0<\sigma<\frac{1}{2} \text {, }
$$

ne segue che is sempre:

$$
v_{1}>v_{\imath} \text {. }
$$


Si spiega questo risultato osservando che una sbarra (mezzo limitato) non subisce variazione di volume quand'è animata da un'onda longitudinale, in quanto - avendo le facce laterali libere - si contrae lateralmente quando sallunga, e si dilata quando si accorcia; le tensioni laterali nulle non generano variazioni nelle dimensioni della sezione retia. Al contrario, il mezzo indefinito non può mutare di dimensioni: occorre quindi una tensione normale magriore per produrre lo stesso allungamemto relativo $\partial u / \partial x$ (con manifesto significato dei simboli).

Ai fini della propagazione delle onde elastiche, un mezzo limitato (sbarra) si comporta quindi come fosse estremamente rigido $(\sigma=0)$.

Pertanto, la velocità $v_{s}$ delle onde trasversali sarà, in esso, espressa da:

$$
v_{s}=\sqrt{\frac{1}{2} \frac{E}{\varrho}} .
$$

Ne viene:

$$
\frac{v_{l}}{v_{s}}=\sqrt{2} .
$$

Possiamo ritenere un concio di una diga come un mezzo limitato. Perturbazioni elastiche, longitudinali e trasversali, condotte da un roncio di una diga devono perciò soddisfare alla [13].

Nelle varie campagne geodinamiche, condotte presso grandi dighe, abbiamo avuto modo di ottenere gran copia di vibrogrammi, rilevati nell'interno dei conci centrali di una diga, in occasione di piccole esplosioni provocate al piede del manufatto. Ia media dei valori ottenuti soddisfa, in modo egregio, alla [13]. Qualehe esempio di onde $l$ ed $s$ è dato dalle Figg. 9, 10, 11, 12 .

In conclusione, in una diga si verificano i seguenti, contrastanti fenomeni.

a) - Dal basso all'alto, lungo un singolo concio, si ha un addensamento di energia verso il coromamento, tanto più marcato quanto maggiore ì l'assottigliamento del concio verso la parte alta. Tale addensamento di energia, che si traduce in un aumento degli spostamenti associati alle onde elastiche, noi definiamo con la qualifica di "effetto di convogliamento".

b) - Alla superficie del piano stradale, con cui una diga ha termine, si manifesta un ulteriore aumento degli spostamenti (analogo 
at quello che si osserva alla superficie di mezzi indefiniti, per le ordinarie onde sismiche). È questo il fenomeno conosciuto sotto la dizione "effetto di superficie"

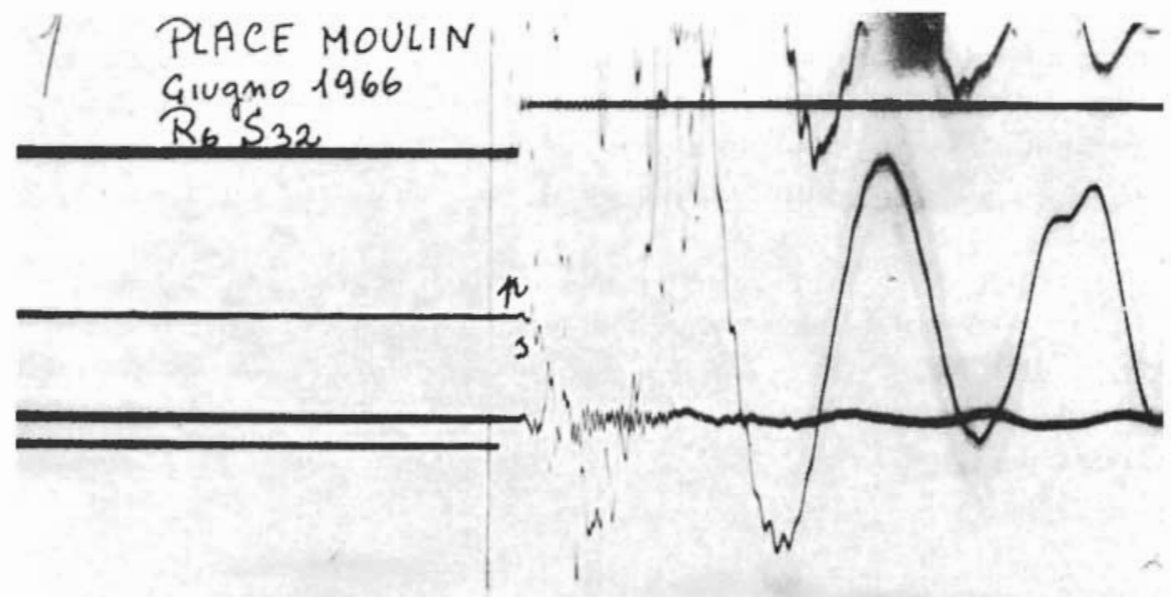

Fig. 9- Esempio di onde longitudinali $(p)$ |corrispondenti ad $(l)$ del testo] e traversali $(x)$ regist rate nel concio di una diga. Le ampie oscillazioni a lungo periodo che seruono, sono dovute alle oscillazioni libere del roncio (vesli paragrafo 4 ).

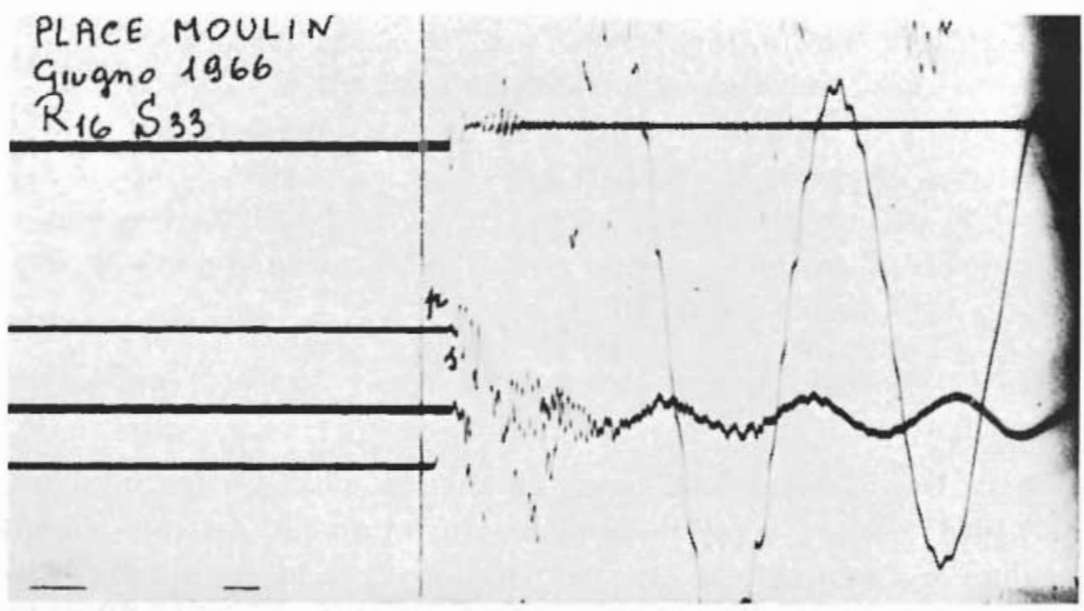

Fig. I0 - Altro esempio come da Fig. 9.

c) - Trasversalmente alla diga, alla stessa quota da destra a sinistra (o viceversa), si verifica invece una sensibile diffusione di energin 
elastica, in corrispondenza delle interposte superficie di discontinuità dinamica fra concio e concio. La progressiva estinzione di energia in questa propagazione trasversa ì da attribuire, nella sua parte pre-

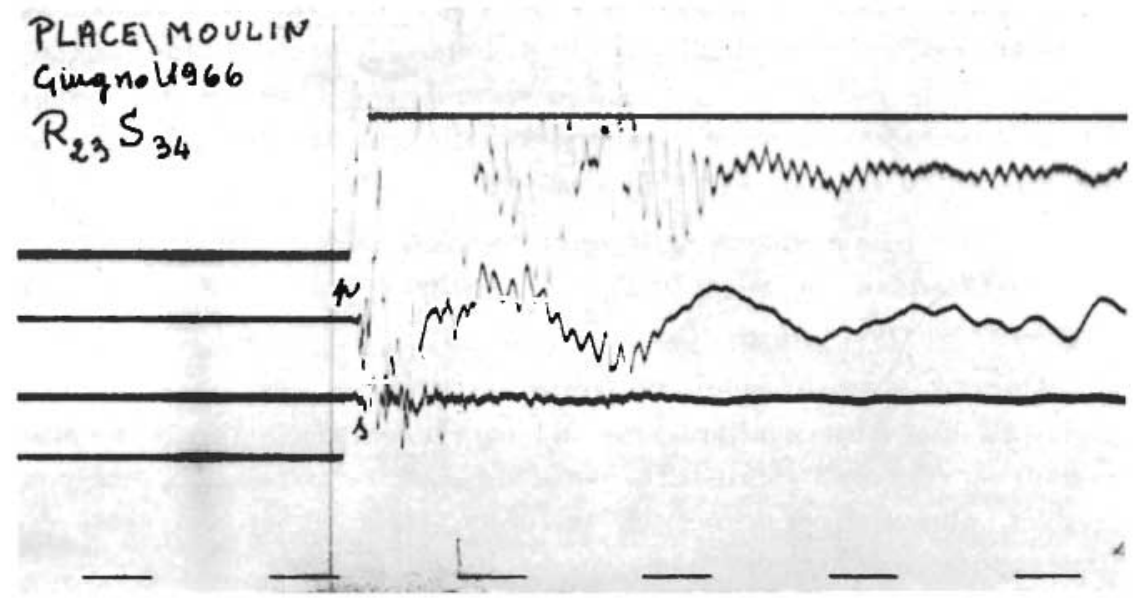

Fig. 11 - Thteriore exempio eome da Fig. 9

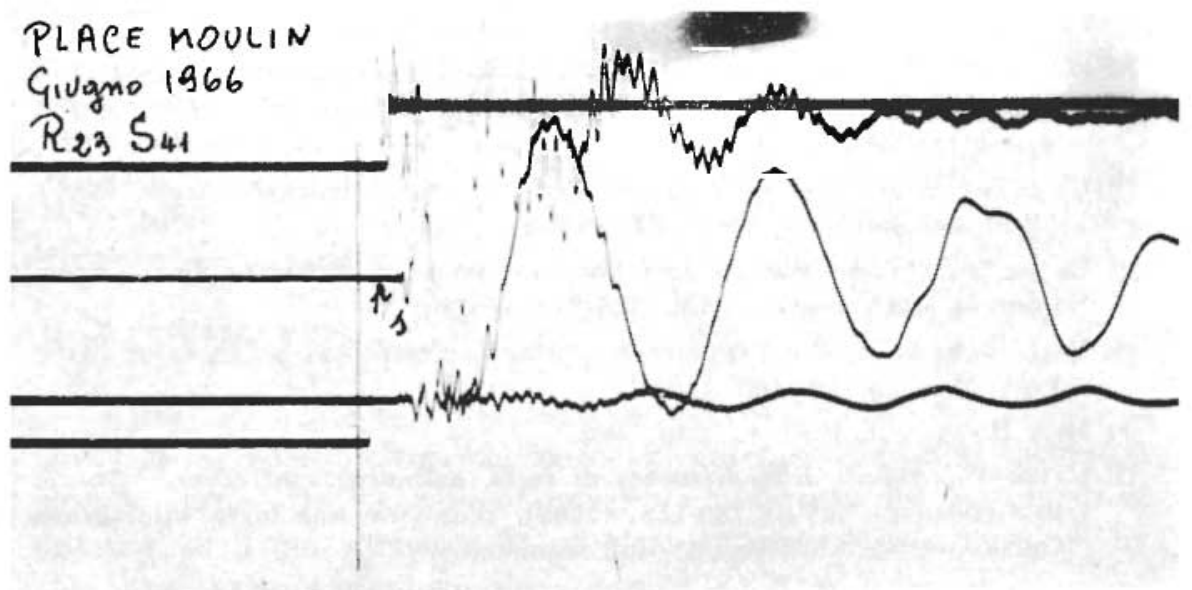

Fir. 12 - Aneora un esempio come da Fig. 9

ponderante, a fenomeni di riflessione in corrispondenza delle superficie arcennate.

d) - OItre a condurre energia elastica, i conci di una diga possono divenire sede di oscillazioni proprie, caratterizzate da periodi d'oscil- 
lazione nettamente superiori a quelli associati alle onde longitudinali e trasversali, condotte dal mezzo.

e) - L'intera diga può entrare in oscillazione libera, nel caso di sollecitazioni capaci di interessarla nella sua totalita, come si verifica in genere nell'apertura degli scarichi di fondo, di mezzo fondo o di superficis. Dette oscillazioni presentano naturalmente $i$ periodi piu elevati, di cui il manufatto è capace (per es. dell'ordine di 111 secondo e mezzo, nel caso della diga di Pieve di ('adore).

f) - I a propagazione delle onde spaziali, longitudinali e trasversali, verificandosi in mezzi definiti, si realizza come se il mezzo fosse estremamente rigido $(\sigma=0)$.

Quanto è stato sopra riassunto, si riferisce alla risposta delle dighe ad una brusca alterazione del campo elastico. Alle lente alterazioni - rilevabili sopra tutto con clinografi - associate a fenomeni termici, climatici, gravimetrici, tettonici, ecc... sono già state dedicate numerose indagini $\left({ }^{8,7,8}\right)$.

\section{BIBLIOGRAFIA}

(1) Calor P., Dispersione delle onde sismiche nell ambito delle altissime frequenze. "Rend. Acc. Naz. Lincei, Classe Scienze fis,, mat., nat, ", serie VIII, XXIV, 3 (1958).

(2) Gutexiser: B., Energy Ratio of Reflected and Refracted Seismic waves. "Bull. Seismol. Soc. Am. ", 34, 85-102, (1944).

$\left.{ }^{3}\right)$ Lanis H., On the Flesure and the Vibrations of a Curned Bar. "Proc. London Math. Soc. ", XIX, 365-376, (1888).

(4) Dex II ARтoG J. P., The Lowest Natural Frequency of Circular Ares. Phil. Mag., 5, 400-408, (1928).

(5) DEN HARTOG: J. P., l. r., pag. 406.

$\left.{ }^{8}\right)$ CALor P., Aspelti della dinamica di rocce, calcestruzzo ed acque. "Anuali di Geofisica n, XV, 2,135-158, (I962)-(eontiene una larga bibliografia sui lavori, compiuti dall' $A$., sull'argomento).

(7) CaLor P., La geodinamica al servizio delle grandi dighe. "Annali di (ieofisica n, XVI, 4, 449.462, (1965).

(8) Caloi P., Spanea M. C., Principali risultati conseguiti durante l'osserva. sione geodinamica, opportunamente extesa nel tempo, di grandi dighe di sbarramento, e loro giustificazioni teoriche. "Annali di Geofisica". XIX, 2. 261.286, (1966). 